\title{
Quantas medidas de pressões respiratórias são necessárias para se obterem medidas máximas em pacientes com tetraplegia?
}

How many maneuvers of respiratory pressures are required to obtain maximal values in patients with quadriplegia?

\author{
¿Cuántas maniobras son necesarias para llegar a las presiones \\ máximas en pacientes con tetraplejía?
}

\author{
Ada Clarice Gastaldi ${ }^{1}$ \\ Getúlio Antonio de Freitas Filho² \\ Ana Paula Manfio Pereira ${ }^{3}$ \\ Janne Marques Silveira ${ }^{4}$
}

\section{RESUMO}

Introdução: pressões inspiratórias (PImax) ou expiratórias (PEmax) máximas constituem um método simples e não-invasivo para avaliação da força de músculos respiratórios e auxiliam na identificação de fraqueza dos músculos respiratórios, presente em diversas doenças e situações clínicas, como a tetraplegia. Objetivo: avaliar o número de manobras necessárias para atingir as pressões máximas em pacientes com tetraplegia. Métodos: oito pacientes com tetraplegia (sete homens), média de idade de $37,8 \pm 11,96$ anos, com diagnóstico de lesão raquimedular cervical completa realizaram 10 medidas de PImax e PEmax nas posições sentada e deitada, totalizando 320 medidas. Os dados foram comparados pelo teste de Wilcoxon $(p<0,05)$. Resultados: os valores obtidos na primeira e na décima medida de PImax na posi-

\section{ABSTRACT}

Introduction: maximum inspiratory (IPmax) and expiratory (EPmax) pressures constitute a simple noninvasive method for evaluation of respiratory muscle strength which helps in the identification of muscle weakness usually present in several diseases and clinical situations, such as quadriplegia. Objective: to assess the number of maneuvers needed to achieve maximum pressures in patients with quadriplegia. Methods: eight quadriplegic patients (seven men) with mean age of $37.8 \pm$ 11.96 years old and presenting with diagnosis of complete spinal cord lesion were submitted to 10 measurements of IPmax and EPmax in both seated and supine positions, totalizing 320 measurements. Data were compared by using the Wilcoxon's test $(p<0.05)$. Results: the $1^{\text {st }}$ and $10^{\text {th }}$ measurements of

\section{RESUMEN}

Introducción: las presiones inspiratorias (PImax) y espiratorias (PEmax) máximas constituyen un método simple y no invasivo para evaluar la fuerza de los músculos respiratorios, y ayudan a identificar la debilidad de los músculos respiratorios presente en diferentes enfermedades y situaciones clínicas, como la tetraplejía. Objetivo: evaluar el número de maniobras necesarias para llegar a las presiones máximas en pacientes con tetraplejía. Métodos: fueron incluidos ocho pacientes con tetraplejía (siete hombres), con edad media de 37,8 $\pm 11,96$ años y diagnóstico de lesión cervical raquis medular completa, a lo que se le realizaron 10 mediciones de PImax y PEmax en posición sentado y acostado, totalizando 320 mediciones. Los datos fueron comparados por el test de Wilcoxon $(p<0,05)$. Resultados: las medidas obtenidas en la primera

\footnotetext{
Departamento de Biomecânica, Medicina e Reabilitação do Aparelho Locomotor - Faculdade de Medicina de Ribeirão Preto da Universidade de São Paulo - USP _ Ribeirão Preto (SP), Brasil.

1 Doutora; Professora do Curso de Fisioterapia do Departamento de Biomecânica, Medicina e Reabilitação do Aparelho Locomotor da Faculdade de Medicina de Ribeirão Preto da Universidade de São Paulo - USP - Ribeirão Preto (SP), Brasil.

2 Mestre; Coordenador do Curso de Fisioterapia do Instituto de Ensino Superior de Rio Verde - IESRIVER - Rio Verde (GO), Brasil.

${ }^{3}$ Mestre; Especialista de Laboratório do Curso de Fisioterapia do Departamento de Biomecânica, Medicina e Reabilitação do Aparelho Locomotor da Faculdade de Medicina de Ribeirão Preto da Universidade de São Paulo - USP - Ribeirão Preto (SP), Brasil.

${ }^{4}$ Mestre; Professor do Curso de Fisioterapia da Universidade de Gurupi - UNIRG - Gurupi (TO), Brasil. 
ção sentada variaram de $74,1 \pm 15,1$ a 74,8 $\pm 19,8 \mathrm{cmH}_{2} \mathrm{O}$ e de PEmax de $32,4 \pm 6,8$ a $32,4 \pm 9,0 \mathrm{cmH}_{2} \mathrm{O}$; na posição deitada, de $76,5 \pm 18,6$ a $91,1 \pm 13,3$ $\mathrm{cmH}_{2} \mathrm{O}(\mathrm{p}<0,05)$ e de $32,5 \pm 5,8$ a $32,9 \pm 5,1 \quad \mathrm{cmH}_{2} \mathrm{O}$, respectivamente. Os resultados das 3 e 5 primeiras medidas com 10 medidas de PImax na posição sentada foram $81,1 \pm 19,5$; $81,5 \pm 18,8$ e $83,0 \pm 18,9 \mathrm{cmH}_{2} \mathrm{O}$ e de PEmax 35, $0 \pm 8,2 ; 35,3 \pm 7,9$ e $36,8 \pm 8,0$ $\mathrm{cmH}_{2} \mathrm{O}$. A PImax na posição deitada foi $90,3 \pm 17,8 ; 94,6 \pm 16,0$ e $97,4 \pm 17,8$ $\mathrm{cmH}_{2} \mathrm{O}(\mathrm{p}<0,05)$ e a PEmax 33,3 $\pm 5,8$; $35,6 \pm 5,4$ e $36,9 \pm 4,9 \mathrm{cmH}_{2} \mathrm{O}$. O maior valor ocorreu a partir da $6^{2}$ medida em $40 \%$ dos testes. Conclusões: para obtenção de valores máximos de pressões respiratórias em pacientes com tetraplegia, é necessária a repetição de ao menos 10 medidas em cada avaliação.
IPmax and EPmax for seated position ranged from $74.1 \pm 15.1$ to $74.8 \pm 19.8$ $\mathrm{cmH}_{2} \mathrm{O}$ and $32.4 \pm 6.8$ to $32.4 \pm 9.0$ $\mathrm{cmH}_{2} \mathrm{O}$, respectively; whereas for supine position such measurements ranged from $76.5 \pm 18.6$ to $91.1 \pm 13.3$ $\mathrm{cmH}_{2} \mathrm{O} \quad(p<0.05)$ and $32.5 \pm 5.8$ to $32.9 \pm 5.1 \mathrm{CmH}_{2} \mathrm{O}$, respectively. The results regarding the $3^{\text {rd }}$ and $5^{\text {th }}$ measurements of IPmax for seated position were $81.1 \pm 19.5,81.5 \pm 18.8$,

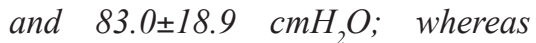
EPmax had 35.0 $\pm 8.2 ; 35.3 \pm 7.9$, and $36.8 \pm 8.0 \mathrm{cmH}_{2} \mathrm{O}$. IPmax values for seated position were 90.3 \pm 17.8 ,

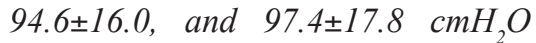
$(p<0.05)$, whereas EPmax had $33.3 \pm 5.8, \quad 35.6 \pm 5.4$, and 36.9 44.9 $\mathrm{cmH}_{2} \mathrm{O}$. The highest value occurred from the $6^{\text {th }}$ measurement in $40 \%$ of the tests. Conclusions: To obtain maximum values for respiratory pressures in quadriplegic patients, it is necessary to repeat the measurements at least 10 times for each evaluation.

KEYWORDS: Quadriplegia;

Respiratory muscles;

Muscle strength; Evaluation;

Manometry y decima medición en posición sentada variaron de 74,1 $\pm 15,1 \mathrm{cmH}_{2} \mathrm{O}$ a $74,8 \pm 19,8 \mathrm{cmH}_{2} \mathrm{O}$ para PImax y de $32,4 \pm 6,8 \mathrm{cmH}_{2} \mathrm{O}$ a 32,4 $\pm 9,0 \mathrm{cmH}_{2} \mathrm{O}$ para PEmax; $y$ en posición acosta-

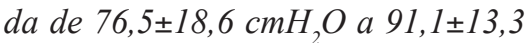
$\mathrm{cmH}_{2} \mathrm{O} \quad(p<0,05) \quad y$ de $32,5 \pm 5,8$

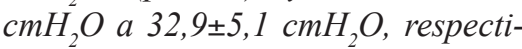
vamente. Los resultados en posición sentada de PImax de las 3 y 5 primeras mediciones de las 10 mediciones fueron 81,1 $19,5 \mathrm{cmH}_{2} \mathrm{O} ; 81,5 \pm 18,8$

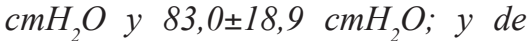
PEmax 35,0 $\pm 8,2 \mathrm{CmH}_{2} \mathrm{O} ; 35,3 \pm 7,9$ $\mathrm{CmH}_{2} \mathrm{O}$ e 36,8 $\pm 8,0 \mathrm{CmH}_{2} \mathrm{O}$. La PImax en posición acostada fue de 90,3 $\pm 17,8 \mathrm{cmH}_{2} \mathrm{O} ; 94,6 \pm 16,0 \mathrm{cmH}_{2} \mathrm{O}$ y 97,4 $\pm 17,8 \mathrm{cmH}_{2} \mathrm{O}(\mathrm{p}<0,05)$ y la PEmax fue de 33,3 $\pm 5,8 \mathrm{cmH}_{2} \mathrm{O}$;

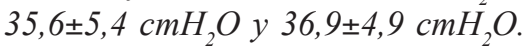
El mayor valor ocurrió a partir de la sexta medida en el $40 \%$ de los testes. Conclusiones: para obtener valores máximos de presiones respiratorios en pacientes con tetraplejía, es necesario repetir por lo menos 10 veces las mediciones en cada evaluación.

DESCRIPTORES: Cuadriplejía; Músculos respiratorios; Fuerza muscular; Evaluación; Manometría

\section{INTRODUÇÃO}

Pressões respiratórias máximas, inspiratórias (PImax) ou expiratórias (PEmax), constituem um método simples e não-invasivo para avaliação da força de músculos respiratórios ${ }^{1}$. Para realização dos testes, o paciente deve gerar a máxima pressão possível contra uma peça bucal bloqueada. As medidas de PImax ou PEmax são frequentemente utilizadas na prática diária de avaliação respiratória e auxiliam na identificação de fraqueza dos músculos respiratórios, presente em diversas doenças e situações clínicas, como a tetraplegia ${ }^{2}$.

O equipamento mais utilizado, denominado manovacuômetro, é capaz de medir a PImax em pressão negativa e a PEmax em pressão positiva. Os modelos de manovacuômetro disponíveis comercialmente limitam-se a valores máximos de 120,150 ou $300 \mathrm{cmH}_{2} \mathrm{O}$, diferindo no nível de precisão $\left(1,4,5\right.$ ou $\left.10 \mathrm{cmH}_{2} \mathrm{O}\right)$.

As diferenças na técnica de medida são apontadas como possíveis causas de resultados divergentes na literatura, dentre outras. Uma das variações relaciona-se ao número de manobras necessárias para se obterem valores máximos. Por se tratar de manobra forçada e dependente de colaboração do avaliado, o aumento do número de medidas tem por objetivo abolir ou minimizar o efeito aprendizado durante os testes.

A American Thoracic Society ${ }^{3}$ recomenda a realização de dez manobras em posição sentada, escolhendo-se o maior valor obtido entre elas, desde que não corresponda à última medida realizada. Apesar da recomendação, alguns estudos utilizam as dez manobras sugeridas ou mais, enquanto outros utilizam três manobras, entre três e cinco manobras aceitáveis, ou ainda cinco manobras ${ }^{4-9}$.

O objetivo deste estudo foi avaliar o número de manobras necessárias para atingir as pressões máximas em pacientes com tetraplegia.

\section{MÉTODOS}

O estudo foi iniciado após aprovação pelo Comitê de Ética em Pesquisa da Instituição. Os voluntários foram esclarecidos com relação ao estudo através de explicações quanto 
ao sistema e seus direitos, e assinaram o termo de consentimento livre e esclarecido.

Foram avaliados 8 pacientes com tetraplegia (sete homens e uma mulher), com média de idade de 37,8 $\pm 11,96$ anos, variando de 19 a 52 anos. Todos tinham diagnóstico de lesão raquimedular completa, variando entre os segmentos de C4 a C7, com tempo médio de lesão de 101,3 meses, com variação de 35 a 318 meses. As lesões foram causadas por acidente automobilístico (4), mergulho (3) e arma de fogo (1).

Como critério de inclusão, o paciente deveria ser portador de lesão medular cervical completa há mais de um ano, em período clinicamente estável.

Foram excluídos aqueles que realizaram fisioterapia respiratória durante o período de coleta de dados, tabagistas ou portadores de doença pulmonar crônica, em estado febril ou com infecções ou viroses no período da coleta ou há menos de 30 dias.

\section{Protocolo}

Foi realizada uma avaliação prévia para coleta de dados e observação dos critérios de inclusão.

No momento do teste, os voluntários incluídos foram instruídos sobre o procedimento e a maneira correta de ser realizado. A motivação era dada durante todo o processo por meio de comando verbal.

As mensurações da PImax e PEmax nas posições sentada e deitada foram feitas através do manovacuômetro analógico, em ordem aleatória.

\section{Medidas das pressões respiratórias}

Os indivíduos realizaram os testes na posição sentada e na posição deitada com o nariz ocluído por um clipe. Foram orientados também a não realizarem refeições por pelo menos duas horas antes dos procedimentos.

Para as medidas de pressão inspiratória máxima, os voluntários foram orientados a realizarem uma expiração máxima, até o volume residual (VR) e, em seguida, realizar um esforço inspiratório máximo no bocal, contra um sistema fechado.

Para as medidas de pressão expiratória máxima, os voluntários foram orientados a realizarem uma inspiração máxima, até a capacidade pulmonar total (CPT) e, em seguida, realizar um esforço expiratório máximo no bocal, contra um sistema fechado.

As pressões foram determinadas durante a realização de esforços com a manutenção da pressão por no mínimo um segundo.

O intervalo das manobras foi de 1 a 2 minutos e, entre um equipamento e outro, de 15 minutos aproximadamente e $^{1,5,8}$.

Foram realizadas 10 medidas para cada variável e em cada posição, totalizando 40 medidas, desde que o maior valor não correspondesse à última medida realizada. Nesse caso, a medida era repetida até a sua estabilização.

O equipamento utilizado foi o manovacuômetro analógico (Marshall Town, MV, 120, USA). O aparelho foi calibrado anteriormente ao início do estudo, em laboratório credenciado segundo recomendações do INMETRO (Instituto Nacional de Metrologia, Normalização e Qualidade Industrial).

As medidas foram realizadas com um bocal rígido com orifício de $1 \mathrm{~mm}$, a fim de evitar influência dos músculos orofaciais e o fechamento da glote, conectado ao manovacuômetro.

O maior valor obtido numa série de medidas de pressões respiratórias é considerado a medida de PImax ou PEmax, por todos os autores da literatura, pois o teste deve refletir o melhor desempenho possível. Para comparações, utilizamos o maior valor obtido nas 3 e 5 primeiras medidas e com o maior valor obtido entre as 10 medidas realizadas.

\section{Análise estatística}

A análise estatística foi realizada por meio do programa a SPSS 16.0 (SPSS, Inc., USA).

Os valores de PImax e PEmax obtidos na posição sentada e deitada foram analisados pelo teste de Friedman para comparação entre as dez medidas obtidas para cada avaliação.

Os valores obtidos com três e cinco medidas foram comparados aos valores obtidos com dez medidas, pelo teste de Wilcoxon, com nível de significância estabelecido em $\mathrm{p}<0,05$.

\section{RESULTADOS}

Foram realizadas 10 medidas para PImax e 10 medidas de PEmax de 8 pacientes, nas posições sentada e deitada, totalizando 320 medidas.

Os valores de PImax e PEmax obtidos na posição sentada estão expressos nas tabelas 1 e 2 . Os valores de PImax variaram de $74,1 \pm 15,1 \mathrm{cmH}_{2} \mathrm{O}$ na primeira medida a $74,8 \pm 19,8 \mathrm{cmH}_{2} \mathrm{O}$ na décima medida, enquanto os valores de PEmax variaram de $32,4 \pm 6,8 \mathrm{cmH}_{2} \mathrm{O}$ na primeira medida a $32,4 \pm 9,0 \mathrm{cmH}_{2} \mathrm{O}$ na décima medida. Essas diferenças não foram estatisticamente significativas.

Os valores de PImax e PEmax obtidos na posição deitada estão expressos nas tabelas 3 e 4 . Os valores de PImax variaram de $76,5 \pm 18,6 \mathrm{cmH}_{2} \mathrm{O}$ na primeira medida a $91,1 \pm 13,3 \mathrm{cmH}_{2} \mathrm{O}$ na décima medida $(\mathrm{p}<0,05)$, enquanto os valores de $\mathrm{PEmax}$ variaram de $32,5 \pm 5,8 \mathrm{cmH}_{2} \mathrm{O}$ na primeira medida a $32,9 \pm 5,1 \mathrm{cmH}_{2} \mathrm{O}$ na décima medida. Essa diferença não foi estatisticamente significativa.

Quando foram comparados os valores médios de PImax na posição sentada obtidos nas 3 primeiras medidas $\left(81,1 \pm 19,5 \mathrm{cmH}_{2} \mathrm{O}\right)$ ou nas 5 primeiras medidas $\left(81,5 \pm 18,8 \mathrm{cmH}_{2} \mathrm{O}\right) \mathrm{com}$ os valores médios obtidos com 10 medidas $\left(83,0 \pm 18,9 \mathrm{cmH}_{2} \mathrm{O}\right)$, essas diferenças não foram estatisticamente significativas. Da mesma forma, quando comparados os valores médios de PEmax na posição sentada obtidos nas 3 primeiras medidas $(35,0 \pm 8,2$ $\left.\mathrm{cmH}_{2} \mathrm{O}\right)$ ou nas 5 primeiras medidas $\left(35,3 \pm 7,9 \mathrm{cmH}_{2} \mathrm{O}\right)$ com os valores médios obtidos com 10 medidas $(36,8 \pm 8,0$ $\mathrm{cmH}_{2} \mathrm{O}$ ), essas diferenças não foram estatisticamente significativas (Figura 1). 
TABELA 1 - Valores individuais das dez medidas de PImax $\left(\mathrm{cmH}_{2} \mathrm{O}\right)$ dos oito pacientes na posição sentada

\begin{tabular}{lccccccccccc}
\hline & \multirow{2}{*}{ Paciente } & $\mathbf{1}$ & $\mathbf{2}$ & $\mathbf{3}$ & $\mathbf{4}$ & $\mathbf{5}$ & $\mathbf{6}$ & $\mathbf{7}$ & $\mathbf{8}$ & $\mathbf{9}$ & $\mathbf{1 0}$ \\
\cline { 2 - 12 } & 1 & 68 & 72 & 56 & 62 & 56 & 48 & 68 & 72 & 66 & 58 \\
& 2 & 49 & 48 & 40 & 52 & 52 & 48 & 40 & 44 & 48 & 44 \\
& 3 & 98 & 89 & 65 & 85 & 84 & 71 & 87 & 68 & 84 & 76 \\
Posição sentada & 4 & 74 & 74 & 96 & 88 & 96 & 88 & 96 & 98 & 92 & 96 \\
& 5 & 80 & 90 & 70 & 86 & 68 & 91 & 72 & 96 & 88 & 91 \\
& 6 & 80 & 72 & 70 & 56 & 67 & 64 & 60 & 66 & 58 & 74 \\
& 7 & 84 & 92 & 104 & 94 & 100 & 102 & 102 & 100 & 100 & 99 \\
& 8 & 60 & 48 & 56 & 56 & 56 & 60 & 64 & 64 & 52 & 60 \\
\hline Média & & 74,1 & 73,1 & 69,6 & 72,4 & 72,4 & 71,5 & 73,6 & 76,0 & 73,5 & 74,8 \\
Desvio padrão & & 15,1 & 17,6 & 21,2 & 17,4 & 18,7 & 20,3 & 20,5 & 20,0 & 19,9 & 19,8 \\
\hline
\end{tabular}

TABELA 2 - Valores individuais das dez medidas de PEmax $\left(\mathrm{cmH}_{2} \mathrm{O}\right)$ dos oito pacientes na posição sentada

\begin{tabular}{lccccccccccc}
\hline & \multicolumn{10}{c}{ Medidas } \\
\cline { 2 - 12 } & Paciente & $\mathbf{1}$ & $\mathbf{2}$ & $\mathbf{3}$ & $\mathbf{4}$ & $\mathbf{5}$ & $\mathbf{6}$ & $\mathbf{7}$ & $\mathbf{8}$ & $\mathbf{9}$ & $\mathbf{1 0}$ \\
\hline & 1 & 28 & 22 & 28 & 24 & 22 & 26 & 22 & 26 & 26 & 24 \\
& 2 & 42 & 39 & 28 & 40 & 32 & 44 & 44 & 36 & 32 & 46 \\
& 3 & 36 & 28 & 28 & 24 & 24 & 24 & 25 & 26 & 26 & 29 \\
Posição sentada & 4 & 38 & 36 & 36 & 36 & 36 & 32 & 36 & 40 & 36 & 32 \\
& 5 & 23 & 19 & 17 & 24 & 20 & 21 & 23 & 24 & 21 & 20 \\
& 6 & 24 & 25 & 27 & 27 & 28 & 30 & 30 & 33 & 34 & 30 \\
& 7 & 36 & 46 & 47 & 45 & 47 & 44 & 44 & 40 & 44 & 44 \\
& 8 & 32 & 39 & 36 & 38 & 34 & 38 & 39 & 38 & 38 & 34 \\
\hline Média & & 32,4 & 31,8 & 30,9 & 32,3 & 30,4 & 32,4 & 32,9 & 32,9 & 32,1 & 32,4 \\
Desvio padrão & & 6,8 & 9,6 & 8,8 & 8,5 & 8,8 & 8,8 & 9,1 & 6,7 & 7,5 & 9,0 \\
\hline
\end{tabular}

TABELA 3 - Valores individuais das dez medidas de PImax $\left(\mathrm{cmH}_{2} \mathrm{O}\right)$ dos oito pacientes na posição deitada

\begin{tabular}{lccccccccccc}
\hline & Paciente & $\mathbf{1}$ & $\mathbf{2}$ & $\mathbf{3}$ & $\mathbf{4}$ & $\mathbf{5}$ & $\mathbf{6}$ & $\mathbf{7}$ & $\mathbf{8}$ & 9 & 10 \\
\cline { 2 - 13 } & 1 & 76 & 96 & 76 & 78 & 96 & 92 & 100 & 80 & 104 & 104 \\
& 2 & 64 & 39 & 44 & 68 & 76 & 72 & 76 & 70 & 78 & 76 \\
& 3 & 76 & 80 & 76 & 84 & 90 & 75 & 80 & 84 & 82 & 92 \\
Posição deitada & 4 & 104 & 100 & 92 & 80 & 96 & 88 & 100 & 100 & 100 & 100 \\
& 5 & 82 & 108 & 90 & 11 & 92 & 102 & 112 & 120 & 102 & 96 \\
& 6 & 56 & 64 & 92 & 94 & 100 & 84 & 76 & 96 & 92 & 88 \\
& 7 & 100 & 100 & 110 & 103 & 115 & 114 & 100 & 106 & 108 & 105 \\
& 8 & 54 & 52 & 68 & 48 & 60 & 70 & 60 & 68 & 64 & 68 \\
\hline Média & & 76,5 & 79,9 & 81,0 & 70,8 & 90,6 & 87,1 & 88,0 & 90,5 & 91,3 & 91,1 \\
Desvio padrão & & 18,6 & 25,5 & 19,8 & 29,3 & 16,4 & 15,3 & 17,5 & 18,2 & 15,3 & 13,3 \\
\hline
\end{tabular}

Quando foram comparados os valores médios de PImax na posição deitada obtidos nas 3 primeiras medidas $\left(90,3 \pm 17,8 \mathrm{cmH}_{2} \mathrm{O}\right)$ com os valores médios obtidos com 10 medidas $\left(97,4 \pm 17,8 \mathrm{cmH}_{2} \mathrm{O}\right)$, essa diferença foi estatisticamente significativa $(\mathrm{p}<0,05)$. Apesar do maior valor obtido com 10 medidas em relação ao valor obtido com as 5 primeiras medidas $\left(94,6 \pm 16,0 \mathrm{cmH}_{2} \mathrm{O}\right)$, essa diferença não foi estatisticamente significativa. Para a PEmax na posição deitada, os valores obtidos com as 3 primeiras medidas $\left(33,3 \pm 5,8 \mathrm{cmH}_{2} \mathrm{O}\right)$ ou com as 5 primeiras medidas $\left(35,6 \pm 5,4 \mathrm{cmH}_{2} \mathrm{O}\right)$ com os valores médios obtidos com 10 medidas $\left(36,9 \pm 4,9 \mathrm{cmH}_{2} \mathrm{O}\right)$, essas diferenças não foram estatisticamente significativas (Figura 1). 
TABELA 4 - Valores individuais das dez medidas de PEmax $\left(\mathrm{cmH}_{2} \mathrm{O}\right)$ dos oito pacientes na posição deitada

\begin{tabular}{lccccccccccc}
\hline & \multicolumn{10}{c}{ Medidas } \\
\cline { 2 - 12 } & Paciente & $\mathbf{1}$ & $\mathbf{2}$ & $\mathbf{3}$ & $\mathbf{4}$ & $\mathbf{5}$ & $\mathbf{6}$ & $\mathbf{7}$ & $\mathbf{8}$ & $\mathbf{9}$ & $\mathbf{1 0}$ \\
\hline & 1 & 36 & 24 & 28 & 30 & 30 & 28 & 30 & 34 & 34 & 35 \\
& 2 & 36 & 36 & 38 & 40 & 36 & 40 & 40 & 40 & 40 & 40 \\
Posição deitada & 3 & 32 & 32 & 28 & 32 & 32 & 34 & 32 & 34 & 32 & 28 \\
& 4 & 28 & 28 & 32 & 40 & 38 & 32 & 36 & 24 & 30 & 32 \\
& 5 & 28 & 25 & 28 & 28 & 28 & 30 & 24 & 26 & 22 & 28 \\
& 6 & 28 & 28 & 28 & 30 & 31 & 28 & 29 & 28 & 26 & 32 \\
& 7 & 44 & 40 & 40 & 36 & 37 & 36 & 44 & 44 & 44 & 40 \\
Média & 8 & 28 & 28 & 28 & 34 & 32 & 32 & 31 & 40 & 36 & 28 \\
Desvio padrão & & 32,5 & 30,1 & 31,3 & 33,8 & 33,0 & 32,5 & 33,3 & 33,8 & 33,0 & 32,9 \\
\hline
\end{tabular}

Entre as 10 medidas realizadas, o maior valor foi obtido a partir da $6^{\mathrm{a}}$ medida em $40 \%$ dos testes.

\section{DISCUSSÃO}

Este estudo demonstrou que, para avaliar as pressões respiratórias máximas em pacientes com tetraplegia, é necessária a realização de no mínimo dez testes para cada medida. Nossos resultados mostram que a utilização de um menor número de medidas ( 3 ou 5) subestima os valores obtidos de PImax e PEmax, pois em $40 \%$ dos testes os maiores valores são obtidos a partir da $6^{\mathrm{a}}$ medida.

A reprodutibilidade do teste foi testada em pacientes normais e em pacientes com doença pulmonar obstrutiva crônica ${ }^{10,11}$, mas persistem questionamentos sobre sua reprodutibilidade em outros grupos de pacientes ${ }^{12}$. Apesar de muitos estudos para determinação das pressões respiratórias máximas e das diferenças metodológicas entre os autores, confirma-se a necessidade de realização das dez manobras, de acordo com a American Thoracic Society ${ }^{3}$ e estudo de Fiz et al..$^{13}$ e Wen et al. ${ }^{14}$.

Fiz et al. ${ }^{13}$ realizaram 20 manobras em pacientes com doença pulmonar obstrutiva crônica e concluíram que os maiores valores foram obtidos entre a $9^{\mathrm{a}}$ e a $10^{\mathrm{a}}$ manobra e que não houve diferença estatística entre a $9^{\mathrm{a}}$ e a $12^{\mathrm{a}}$, enquanto Wen et al. ${ }^{14}$ realizaram 20 manobras em pacientes adultos e crianças com diversas doenças, concluindo que, em $65 \%$ dos testes, os maiores valores eram atingidos depois da $10^{\mathrm{a}}$ medida.

Os resultados reforçam o possível efeito aprendizado, pois é improvável que os músculos respiratórios aumentem a sua força em alguns minutos, durante o período necessário para a realização dos testes. Ainda que os valores obtidos após dez medidas possam ser questionados, certamente eles representam ou se encontram mais próximos aos valores máximos. Em nosso estudo, utilizamos como critério a repetição de um ou mais testes quando o maior valor ocorresse na décima medida.

Os primeiros estudos que avaliaram a função dos músculos respiratórios e estabeleceram padrões para as medidas de pressões respiratórias foram os estudos

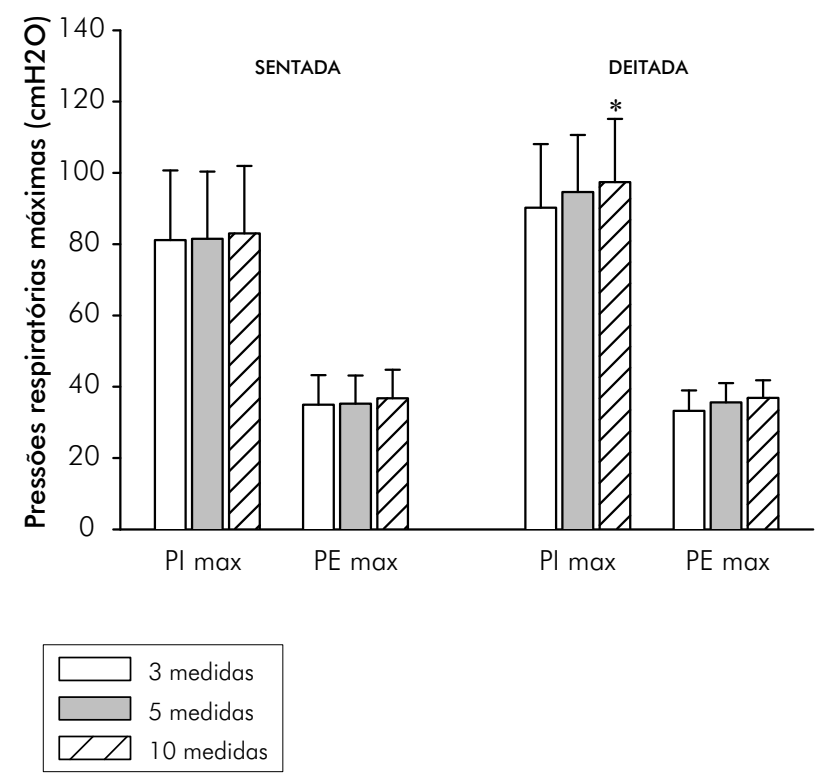

Figura 1

Plmax e PEmax nas posições sentada e deitada dos valores máximos obtidos com 3, 5 e 10 repetições.

realizados por Ringqvist ${ }^{15}$ e por Black e Hyatt ${ }^{4}$, que propuseram valores de normalidade. Ringqvist ${ }^{15}$ utilizou uma média de 10 manobras por teste enquanto Black e Hyatt ${ }^{4}$ utilizaram o maior valor entre duas manobras tecnicamente aceitáveis, obtendo resultados 10 a 20 $\mathrm{cmH}_{2} \mathrm{O}$, menores que os de Ringqvist ${ }^{15}$. No presente estudo, as medidas de PImax obtidas na posição deitada mostram uma diferença, em média, de $15 \mathrm{cmH}_{2} \mathrm{O}$ quando se comparam os resultados obtidos na $1^{\mathrm{a}} \mathrm{com}$ os obtidos na $10^{\mathrm{a}}$ medida.

Enrigh et al. ${ }^{7}$ estudaram pacientes jovens e realizaram apenas cinco manobras. Observaram que o maior valor foi obtido da quinta manobra. No entanto, Wagener ${ }^{16}$ observou que a pressão inspiratória máxima determinada pela melhor de 5 manobras foi 5\% menor que a PImax determinada por 20 manobras. 
Apesar da recomendação, diversos estudos que utilizaram PImax e PEmax para avaliação em diferentes grupos de pacientes utilizaram cinco ou menos medidas. Existem outros estudos ${ }^{4-9,17-20}$ que utilizam de duas a cinco manobras reprodutíveis que não diferem mais de $10 \%$ entre si.

Vale ressaltar que os valores de referência mais utilizados mundialmente foram propostos por Black e Hyatt, que, como já foi dito, utilizaram o maior valor obtido entre duas manobras tecnicamente aceitáveis e, para a população brasileira, utiliza-se a equação proposta por Nedder et al. ${ }^{8}$, cujos resultados foram obtidos com apenas cinco medidas.

Embora as recomendações sejam válidas para PImax e PEmax, a discussão da literatura concentra-se nas medidas de PImax e não são conhecidas comparações para os dados de PEmax.

Em nosso estudo, a diferença foi mais acentuada nas medidas realizadas em posição deitada, que apresenta valores maiores de PImax. O estudo de Wen et al. ${ }^{14}$, que utilizou 20 medidas e comparou os maiores valores iniciais denominados "short MIP", com os maiores valores, denominados "long MIP", pois o maior valor ocorreu em $65 \%$ dos casos após a décima medida, mostram que a diferença entre "short MIP" e "long MIP" foi maior para o grupo com valores de PImax maior que $90 \mathrm{cmH}_{2} \mathrm{O}$. Os autores não discutem esse achado, mas é possível que a diferença esperada seja mais bem expressa em uma medida relativa, como o percentual, do que em valores absolutos.

Outro aspecto interessante discutido por Wen et al. ${ }^{14}$ refere-se a um efeito aprendizado que é importante durante a realização das manobras, mas não é retido pelo paciente de uma semana para outra. Os autores recomendam que mesmo pacientes que já tenham realizado a medida de PImax previamente sejam tratados como inexperientes.

Recente revisão sistemática da literatura discute as divergências metodológicas entre os diversos autores e a forma como elas afetam os valores previstos de PImax. Curiosamente, o número de repetições da medida não é um tópico discutido pelos autores ${ }^{21}$.

Outros estudos que tenham realizado medidas com pacientes tetraplégicos. Nesse grupo, no qual se esperado que as medidas na posição sentada resultem em menores medidas de PImax ${ }^{6,22-24}$, o desempenho durante a medida não se modificou significativamente. Mais interessante ainda é a não-modificação das medidas de PEmax, cujos valores estão bem abaixo da normalidade nesses pacientes.

Assim, concluímos que, para obtenção de valores máximos de pressões respiratórias em pacientes com tetraplegia, é necessária a repetição de ao menos dez medidas.

\section{REFERÊNCIAS}

1. Souza RB. Pressões respiratórias estáticas máximas. J Pneumol. 2002;28(3 Suppl):155-65.

2. Laghi F, Tobin MJ. Disorders of the respiratory muscles. Am J Respir Crit Care Med. 2003;168(1):10-48.

3. American Thoracic Society/European Respiratory Society. ATS/ERS statement on respiratory muscle testing. Am J Respir Crit Care Med. 2002;166(4):518-24.

4. Black LF, Hyatt RE. Maximal respiratory pressures: normal values and relationship to age and sex. Am Rev Respir Dis. 1969;99(5):696-702.

5. Bruschi C, Cerveri I, Zoia MC, Fanfulla F, Fiorentini M, Casali L, et al. Reference values of maximal respiratory mouth pressures: a population-based study. Am Rev Respir Dis. 1992;146(3):790-3.

6. Fiz JA, Texido A, Izquierdo J, Ruiz J, Roig J, Morera J. Postural variation of the maximum inspiratory and expiratory pressures in normal subjects. Chest. 1990;97(2):313-4.

7. Enright PL, Kronmal RA, Manolio, Teri A, Schenker Marc B, Hyatt Robert E. Respiratory muscle strenght in the elderly. Correlates and reference values. Respir Crit Care Med. 1994;149(2):430-8.
8. Neder JA, Andreoni S, Lerario MC, Nery LE. Reference values for lung function test. II Maximal respiratory pressures and voluntary ventilation. Braz J Med Biol Res. 1999;32(6):71927.

9. Tully K, Koke K, Garshick E, Lieberman SL, Tun CG, Brown R. Maximal Expiratory Pressures in Spinal Cord Injury Using Two Mouthpieces. Chest. 1997;112(1):1136.

10.Larson JL, Covery MK, Vitalo CA, Alex CG, Patel M, Kim MJ. Maximal inspiratory pressure: learning effect and test-retes reliability in patients with chronic obstructive pulmonary disease. Chest. 1993;104(2):448-53.

11.Aldrich T, Spiro P. Maximal inspiratory pressure: does reprodutibility indicate full effort? Thorax. 1995;50(1):40-3.

12.Multz AS, Aldrich TK, Prezant DJ, Karpel JP, Hendler JM. Maximal inspiratory pressure is not a reliable test of inspiratory muscle strength in mechanically ventilate patients. Am Rev Respir Dis. 1990;142(2):529-32.
13.Fiz JA, Montserrat JM, Picado C, Plaza V, A Agusti-vidal. How many manoeuvres shold be done to measure maximal inspiratory mouth pressure in patients with chronic obstruction? Thorax. 1989;44(5):419-21.

14. Wen AS, Woo MS, Keens TG. How many maneuvers are required to measure maximal inspiratory pressure accurately? Chest. 1997;111(3):802-7.

15.Ringqvist, $\mathrm{T}$. The ventilatory capacity in healthy subjects: An analysis of casual factors with special reference to the respiratory forces. Sacnd J Clin Lab Invest Suppl. 1966;88:5-179.

16. Wagener J, Hibbert M, Landau L. Maximal respiratory pressures in children. Am Rev Respir Dis. 1984;129(5):873-75.

17.Parreira VF, França DC, Zampa CC, Fonseca MM, Tomich GM, Britto RR. Pressões respiratórias máximas: valores encontrados e preditos em indivíduos saudáveis. Rev Bras Fisioter. 2007;11(5):361-8.

18. Wilson SH, Cooke NT, Edwards RH, Spiro SG. Predicted normal values for maximal respiratory pressures in caucasian adults and children. Thorax. 1984;39(7):535-8. 
19.Camelo Jr JS, Terra JT, Manço JC. Pressões respiratórias máximas em adultos normais. J Pneumol. 1985;11(4):181-4.

20.Harik-Khan RI, WiseRA, Fozard J. Determinants of maximal inspiratory pressure. The Baltimore Longitudinal Study of Aging. Am J Respir Crit Care Med. 1998;158(5):1459-64.

21.Evans JA, Whitelaw WA. The assessment of maximal respiratory mouth pressures in adults. Respir Care. 2009;54(10):1348-59.
22. Boaventura CM, Silveira JM, Santos PR, Gastaldi AC. Força da musculatura respiratória de pacientes tetraplégicos sentados e em supino. Rev Fisioter Univ São Paulo. 2004;11(2):70-6.

23. Boaventura CM, Gastaldi AC, Silveira JM, Guimarães RC, Lima LC. Effect of an abdominal binder on the efficacy of respiratory muscles inseated and supine tetraplegic patients. Physiotherapy. 2003;89(5):290-95.

24.Tully K, Koke K, Garshick E, Lieberman SL, Tun CG, Brown R. Maximal expiratory pressures in spinal cord injury using two mouthpieces. Chest. 1997;112(1):113-6.

\section{Correspondência}

Ada Clarice Gastaldi

Departamento de Biomecânica, Medicina e Reabilitação do Aparelho Locomotor da Faculdade de Medicina de Ribeirão Preto da Universidade de São Paulo.

Avenida Bandeirantes, 3.900

CEP: 14049-900 - Ribeirão Preto (SP), Brasil

Tel./fax: (16) 3602-4413

E-mail:ada@fmrp.usp.br 